\title{
On $q$-analogue of a complex summation-integral type operators in compact disks
}

\author{
Ravi P Agarwal ${ }^{1}$ and Vijay Gupta ${ }^{2^{*}}$
}

\author{
* Correspondence: \\ vijaygupta2001@hotmail.com \\ ${ }^{2}$ School of Applied Sciences, Netaji \\ Subhas Institute of Technology, \\ Sector 3 Dwarka, New Delhi- \\ 110078, India \\ Full list of author information is \\ available at the end of the article
}

\begin{abstract}
Recently, Gupta and Wang introduced certain q-Durrmeyer type operators of real variable $x \in[0,1]$ and studied some approximation results in the case of real variables. Here we extend this study to the complex variable for analytic functions in compact disks. We establish the quantitative Voronovskaja type estimate. In this way, we put in evidence the over convergence phenomenon for these $q^{-}$ Durrmeyer polynomials; namely, the extensions of approximation properties (with quantitative estimates) from the real interval $[0,1]$ to compact disks in the complex plane. Some of these results for $q=1$ were recently established in Gupta-Yadav.

Mathematical subject classification (2000): 30E10; 41A25.
\end{abstract}

Keywords: Complex q-Durrmeyer operators, q-integer, q-factorial, q-Beta function, exact order of approximation, quantitative Voronovskaja-type asymptotic formula

\section{Introduction}

In the recent years applications of $q$-calculus in the area of approximation theory and number theory is an active area of research. Several researchers have proposed the $q$ analogue of exponential, Kantorovich and Durrmeyer type operators. Also Kim $[1,2]$ used $q$-calculus in area of number theory. Recently, Gupta and Wang [3] proposed certain $q$-Durrmeyer operators in the case of real variables. The aim of the present article is to extend approximation results for such $q$-Durrmeyer operators to the complex case. The main contributions for the complex operators are due to Gal; in fact, several important results have been complied in his recent monograph [4]. Also very recently, Gal and Gupta [5-7] have studied some other complex Durrmeyer type operators, which are different from the operators considered in the present article.

We begin with some notations and definitions of $q$-calculus: For each nonnegative integer $k$, the $q$-integer $[k]_{q}$ and the $q$-factorial $[k]_{q}$ ! are defined by

$$
[k]_{q}:=\left\{\begin{array}{lr}
\left(1-q^{k}\right) /(1-q), & q \neq 1 \\
k, & q=1,
\end{array}\right.
$$

\section{Springer}

(C) 2012 Agarwal and Gupta; licensee Springer. This is an Open Access article distributed under the terms of the Creative Commons Attribution License (http://creativecommons.org/licenses/by/2.0), which permits unrestricted use, distribution, and reproduction in any medium, provided the original work is properly cited. 
and

$$
[k]_{q} !:=\left\{\begin{array}{lr}
{[k]_{q}[k-1]_{q} \cdots[1]_{q^{\prime}}} & k \geq 1 \\
1, & k=0
\end{array}\right.
$$

respectively. For the integers $n, k, n \geq k \geq 0$, the $q$-binomial coefficients are defined by

$$
\left[\begin{array}{l}
n \\
k
\end{array}\right]_{q}:=\frac{[n]_{q} !}{[k]_{q} ![n-k]_{q} !}
$$

In this article, we shall study approximation results for the complex $q$-Durrmeyer operators (introduced and studied in the case of real variable by Gupta-Wang [3]), defined by

$$
M_{n, q}(f ; z)=[n+1]_{q} \sum_{k=1}^{n} q^{1-k} p_{n, k}(q ; z) \int_{0}^{1} f(t) p_{n, k-1}(q ; q t) d_{q} t+f(0) p_{n, 0}(q ; z)
$$

where $z \in \mathbb{C}, n=1,2, \ldots ; q \in(0,1)$ and $(a-b)_{q}^{m}=\Pi_{j=0}^{m-1}\left(a-q^{j} b\right), q$-Bernstein basis functions are defined as

$$
p_{n, k}(q ; z):=\left[\begin{array}{l}
n \\
k
\end{array}\right]_{q} z^{k}(1-z)_{q}^{n-k}
$$

also in the above $q$-Beta functions [8] are given as

$$
B_{q}(m, n)=\int_{0}^{1} t^{m-1}(1-q t)_{q}^{n-1} d_{q} t, m, n>0 .
$$

Throughout the present article we use the notation $D_{R}=\{z \in \mathbb{C}:|z|<R\}$ and by $H$ $\left(D_{R}\right)$, we mean the set of all analytic functions on $f: D_{R} \rightarrow \mathbb{C}$ with $f(z)=\sum_{k=0}^{\infty} a_{k} z^{k}$ for all $z \in D_{R}$. The norm ||$f \|_{r}=\max \{|f(z)|:|z| \leq r\}$. We denote $\pi_{p, n}(q ; z)=M_{n, q}\left(e_{p}\right.$; $z$ ) for all $e_{p}=t^{p}, p \in \mathbb{N} \cup\{0\}$.

In what follows, we shall study the approximation properties of the operators $M_{n, q}(f$; $z$ ), which is extension of the results studied in [10]. Further, for these operators we will estimate an upper bound, a quantitative Voronovskaja-type asymptotic formula, and exact order of approximation on compact disks.

\section{Basic results}

To prove the results of following sections, we need the following basic results.

Lemma 1. Let $q \in(0,1)$. Then, $\pi_{m, n}(q ; z)$ is a polynomial of degree $\leq \min (m, n)$, and

$$
\pi_{m, n}(q ; z)=\frac{[n+1]_{q} !}{[n+m+1]_{q} !} \sum_{s=1}^{m} c_{s}(m)[n]_{q}^{s} B_{n, q}\left(e_{s} ; z\right),
$$

where $c_{s}(m) \geq 0$ are constants depending on $m$ and $q$, and $B_{n, q}(f ; z)$ is the q-Bernstein polynomials given by $B_{n, q}(f ; z)=\sum_{k=0}^{n} p_{n, k}(q ; z) f\left([k]_{q} /[n]_{q}\right)$. 
Proof. By definition of $q$-Beta function, with $B_{q}(m, n)=\frac{[m-1]_{q} ![n-1]_{q} !}{[m+n-1]_{q} !}$, we have

$$
\begin{gathered}
\pi_{m, n}(q ; z)=[n+1]_{q} \sum_{k=1}^{n} q^{1-k} p_{n, k}(q ; z) \int_{0}^{1} p_{n, k-1}(q ; q t) t^{m} d_{q} t \\
=[n+1]_{q} \sum_{k=1}^{n} q^{1-k} p_{n, k}(q ; z) \int_{0}^{1}\left[\begin{array}{c}
n \\
k-1
\end{array}\right]_{q}(q t)^{k-1}(1-q t)_{q}^{n-k+1} t^{m} d_{q} t \\
=[n+1]_{q} \sum_{k=1}^{n} p_{n, k}(q ; z) \frac{[n]_{q} !}{[k-1]_{q} ![n-k+1]_{q} !} B_{q}(k+m, n-k+2) \\
=\frac{[n+1]_{q} !}{[n+m+1]_{q} !} \sum_{k=1}^{n} p_{n, k}(q ; z) \frac{[k+m-1]_{q} !}{[k-1]_{q} !} .
\end{gathered}
$$

For $m=1$, we find

$$
\begin{gathered}
\pi_{1, n}(q ; z)=\frac{[n+1]_{q} !}{[n+2]_{q} !} \sum_{k=1}^{n} p_{n, k}(q ; z)[k]_{q}=\frac{1}{[n+2]_{q}} \sum_{k=0}^{n} p_{n, k}(q ; z)[n]_{q} \frac{[k]_{q}}{[n]_{q}} \\
=\frac{1}{[n+2]_{q}} \sum_{s=1}^{1}[n]_{q}^{s} B_{n, q}\left(e_{s} ; z\right),
\end{gathered}
$$

thus the result is true for $m=1$ with $c_{1}(1)=1>0$.

Next for $m=2$, with $[k+1]_{q}=1+q[k]_{q}$, we get

$$
\begin{gathered}
\pi_{2, n}(q ; z)=\frac{[n+1]_{q} !}{[n+3]_{q} !} \sum_{k=0}^{n} p_{n, k}(q ; z)\left(1+q[k]_{q}\right)[k]_{q}=\frac{[n+1]_{q} !}{[n+3]_{q} !}\left[[n]_{q} B_{n, q}\left(e_{1} ; z\right)+q[n]_{q}^{2} B_{n, q}\left(e_{2} ; z\right)\right] \\
=\frac{[n+1]_{q} !}{[n+3]_{q} !} \sum_{s=1}^{2} c_{s}(2)[n]_{q}^{s} B_{n, q}\left(e_{s} ; z\right),
\end{gathered}
$$

thus the result is true for $m=2$ with $c_{1}(2)=1>0, c_{2}(2)=q>0$.

Similarly for $m=3$, using $[k+2]_{q}=[2]_{q}+q^{2}[k]_{q}$ and $[k+1]_{q}=1+q[k]_{q}$ we have

$$
\pi_{3, n}(q ; z)=\frac{[n+1]_{q} !}{[n+4]_{q} !} \sum_{s=1}^{3} c_{s}(3)[n]_{q}^{s} B_{n, q}\left(e_{s} ; z\right)
$$

where $c_{1}(3)=[2]_{q}>0, c_{2}(3)=2 q^{2}+q>0$, and $c_{3}(3)=q^{3}>0$.

Continuing in this way the result follows immediately for all $m \in N$. $\square$

Lemma 2. Let $q \in(0,1)$. Then, for all $m, n \in \mathbb{N}$, we have the inequality

$$
\frac{[n+1]_{q} !}{[n+m+1]_{q} !} \sum_{s=1}^{m} c_{s}(m)[n]_{q}^{s} \leq 1
$$

Proof. By Lemma 1, with $e_{m}=t^{m}$, we have

$$
\pi_{m, n}(q ; 1)=\frac{[n+1]_{q} !}{[n+m+1]_{q} !} \sum_{s=1}^{m} c_{s}(m)[n]_{q}^{s} B_{n, q}\left(e_{s} ; 1\right)=\frac{[n+1]_{q} !}{[n+m+1]_{q} !} \sum_{s=1}^{m} c_{s}(m)[n]_{q}^{s} .
$$

Also

$$
p_{n, k}(q ; z)=\left[\begin{array}{l}
n \\
k
\end{array}\right]_{q} z^{k}(1-z)(1-q z)\left(1-q^{2} z\right) \ldots\left(1-q^{n-k-1} z\right) .
$$


It immediately follows that $p_{n, k}(q ; 1)=0, k=0,1,2, \ldots, n-1$ and $p_{n, n}(q ; 1)=1$.

Thus, we obtain

$$
\begin{aligned}
\pi_{m, n}(q ; 1) & =[n+1]_{q} p_{n, n}(q ; 1) q^{1-n} \int_{0}^{1} p_{n, n-1}(q ; q t) t^{m} d_{q} t \\
& =[n+1]_{q} \int_{0}^{1}[n]_{q} t^{n+m-1}(1-q t) d_{q} t \\
& =[n+1]_{q}[n]_{q}\left[\frac{t^{n+m}}{[n+m]_{q}}-q \frac{t^{n+m+1}}{[n+m+1]_{q}}\right]_{0}^{1} \\
& =\frac{[n+1]_{q}[n]_{q}}{[n+m]_{q}[n+m+1]_{q}} \leq 1 .
\end{aligned}
$$

Corollary 1. Let $r \geq 1$ and $q \in(0,1)$. Then, for all $m, n \in \mathbb{N} \cup\{0\}$ and $|z| \leq r$ we have $\left|\pi_{m, n}(q ; z)\right| \leq r^{m}$.

Proof. By using the methods Gal [4], p. 61, proof of Theorem 1.5.6, we have $\mid B_{n, q}\left(e_{s}\right.$; $z) \mid \leq r^{s}$, By Lemma 2 and for all $m \in \mathbb{N}$ and $|z| \leq r$,

$$
\begin{aligned}
\left|\pi_{m, n}(q ; z)\right| & \leq \frac{[n+1]_{q} !}{[n+m+1]_{q} !} \sum_{s=1}^{m} c_{s}(m)[n]_{q}^{s}\left|B_{n, q}\left(e_{s} ; z\right)\right| \\
& \leq \frac{[n+1]_{q} !}{[n+m+1]_{q} !} \sum_{s=1}^{m} c_{s}(m)[n]_{q}^{s} r^{s} \leq r^{m} .
\end{aligned}
$$

$\square$

Lemma 3. Let $q \in(0,1)$ then for $z \in \mathbb{C}$, we have the following recurrence relation:

$$
\pi_{p+1, n}(q ; z)=\frac{q^{p} z(1-z)}{[n+p+2]_{q}} D_{q} \pi_{p, n}(q ; z)+\frac{q^{p}[n]_{q} z+[p]_{q}}{[n+p+2]_{q}} \pi_{p, n}(q ; z) .
$$

Proof. By simple computation, we have

$$
z(1-z) D_{q}\left(p_{n, k}(q ; z)\right)=\left([k]_{q^{-}}[n]_{q^{\prime}} z\right) p_{n, k}(q ; z),
$$

and

$$
t(1-q t) D_{q}\left(p_{n, k-1}(q ; q t)\right)=\left([k-1]_{q}-[n]_{q} q t\right) p_{n, k-1}(q ; q t) .
$$

Using these identities, it follows that

$$
\begin{gathered}
z(1-z) D_{q}\left(\pi_{p, n}(q ; z)\right)=[n+1]_{q} \sum_{k=1}^{n} q^{1-k}\left([k]_{q}-[n]_{q} z\right) p_{n, k}(q ; z) \int_{0}^{1} p_{n, k-1}(q ; q t) t^{p} d_{q} t \\
=[n+1]_{q} \sum_{k=1}^{n} q^{1-k} p_{n, k}(q ; z) \int_{0}^{1}\left(1+q[k-1]_{q}-[n]_{q} q^{2} t+[n]_{q} q^{2} t\right) p_{n, k-1}(q ; q t) t^{p} d_{q} t \\
-z[n]_{q}[n+1]_{q} \sum_{k=1}^{n} q^{1-k} p_{n, k}(q ; z) \int_{0}^{1} p_{n, k-1}(q ; q t) t^{p} d_{q} t \\
=q[n+1]_{q} \sum_{k=1}^{n} q^{1-k} p_{n, k}(q ; z) \int_{0}^{1}\left(D_{q} p_{n, k-1}(q ; q t)\right) t(1-q t) t^{p} d_{q} t \\
+\pi_{p, n}(q ; z)+[n]_{q} q^{2} \pi_{p+1, n}(q ; z)-z[n]_{q} \pi_{p, n}(q ; z) .
\end{gathered}
$$


Let us denote $\delta(t)=\frac{t}{q}(1-t)\left(\frac{t}{q}\right)^{p}=\frac{1}{q^{p+1}}\left(t^{p+1}-t^{p+2}\right)$. Then, the last $q$-integral becomes

$$
\begin{gathered}
\int_{0}^{1} D_{q}\left(p_{n, k-1}(q ; q t)\right) t(1-q t) t^{p} d_{q} t=\int_{0}^{1} D_{q}\left(p_{n, k-1}(q ; q t)\right) \delta(q t) d_{q} t \\
=\left.\delta(t) p_{n, k-1}(q ; q t)\right|_{0} ^{1}-\int_{0}^{1} p_{n, k-1}(q ; q t) D_{q} \delta(t) d_{q} t \\
=-q^{-p-1} \int_{0}^{1} p_{n, k-1}(q ; q t) D_{q}\left(t^{p+1}-t^{p+2}\right) d_{q} t \\
=-q^{-p-1}[p+1]_{q} \int_{0}^{1} p_{n, k-1}(q ; q t) t^{p} d_{q} t \\
+q^{-p-1}[p+2]_{q} \int_{0}^{1} p_{n, k-1}(q ; q t) t^{p+1} d_{q} t
\end{gathered}
$$

and hence

$$
\begin{gathered}
z(1-z) D_{q} \pi_{p, n}(q ; z)=-q^{-p}[p+1]_{q} \pi_{p, n}(q ; z)+q^{-p}[p+2]_{q} \pi_{p+1, n}(q ; z) \\
+\pi_{p, n}(q ; z)+[n]_{q} q^{2} \pi_{p+1, n}(q ; z)-z[n]_{q} \pi_{p, n}(q ; z) .
\end{gathered}
$$

Therefore,

$$
\begin{gathered}
\pi_{p+1, n}(q ; z)=\frac{z(1-z)}{q^{-p}[p+2]_{q}+[n]_{q} q^{2}} D_{q} \pi_{p, n}(q ; z)+\frac{[n]_{q} z+q^{-p}[p+1]_{q}-1}{q^{-p}[p+2]_{q}+[n]_{q} q^{2}} \pi_{p, n}(q ; z) \\
=\frac{q^{p} z(1-z)}{[p+2]_{q}+[n]_{q} q^{p+2}} D_{q} \pi_{p, n}(q ; z)+\frac{q^{p}[n]_{q} z+[p]_{q}}{[p+2]_{q}+[n]_{q} q^{p+2}} \pi_{p, n}(q ; z) .
\end{gathered}
$$

Finally, using the identity $[p+2]_{q}+[n]_{q} q^{p+2}=[n+p+2]_{q}$, we get the required recurrence relation. $\square$

\section{Upper bound}

If $P_{m}(z)$ is a polynomial of degree $m$, then by the Bernstein inequality and the complex mean value theorem, we have

$$
\left|D_{q} P_{m}(z)\right| \leq\left\|P_{m}^{\prime}\right\|_{r} \leq \frac{m}{r}\left\|P_{m}\right\|_{r} \text { for all }|z| \leq r .
$$

The following theorem gives the upper bound for the operators (1.1).

Theorem 1. Let $f(z)=\sum_{p=0}^{\infty} a_{p} z^{p}$ for all $|z|<R$ and let $1 \leq r \leq R$, then for all $|z| \leq r$, $q \in(0,1)$ and $n \in \mathbb{N}$,

$$
\left|M_{n, q}(f ; z)-f(z)\right| \leq \frac{K_{r}(f)}{[n+2]_{q}}
$$

where $K_{r}(f)=(1+r) \sum_{p=1}^{\infty}\left|a_{p}\right| p(p+1) r^{p-1}<\infty$. 
Proof First, we shall show that $M_{n, q}(f ; z)=\sum_{p=0}^{\infty} a_{p} \pi_{p, n}(q ; z)$. If we denote $f_{m}(z)=\sum_{j=0}^{m} a_{j} z^{j},|z| \leq r$ with $m \in \mathbb{N}$, then by the linearity of $M_{n, q}$, we have

$$
M_{n, q}\left(f_{m} ; z\right)=\sum_{p=0}^{m} a_{p} \pi_{p, n}(q ; z)
$$

Thus, it suffice to show that for any fixed $n \in \mathbb{N}$ and $|z| \leq r$ with $r \geq 1, \lim _{m \rightarrow \infty} M_{n, q}$ $\left(f_{m}, z\right)=M_{n, q}(f ; z)$. But this is immediate from $\lim _{m \rightarrow \infty}|| f_{m}-\left.f\right|_{r}=0$ and by the inequality

$$
\begin{aligned}
& -M_{n, q}\left(f_{m} ; z\right)-M_{n, q}(f ; z)- \\
& \leq\left|f_{m}(0)-f(0)\right| .\left|(1-z)^{n}\right|+[n+1]_{q} \sum_{k=1}^{n}\left|p_{n, k}(q ; z)\right| q^{1-k} \int_{0}^{1} p_{n, k-1}(q, q t)\left|f_{m}(t)-f(t)\right| d_{q} t \\
& \leq C_{r, n}\left\|f_{m}-f\right\|_{r},
\end{aligned}
$$

where

$$
C_{r, n}=(1+r)^{n}+[n+1]_{q} \sum_{k=1}^{n}\left[\begin{array}{l}
n \\
k
\end{array}\right]_{q}(1+r)^{n-k} r^{k} \int_{0}^{1} p_{n, k-1}(q ; q t) d_{q} t .
$$

Since, $\pi_{0, n}(q ; z)=1$, we have

$$
\left|M_{n, q}(f ; z)-f(z)\right| \leq \sum_{p=1}^{\infty}\left|a_{p}\right| .\left|\pi_{p, n}(q ; z)-e_{p}(z)\right| .
$$

Now using Lemma 3, for all $p \geq 1$, we find

$$
\begin{aligned}
\pi_{p, n}(q ; z)-e_{p}(z) & =\frac{q^{p-1} z(1-z)}{[n+p+1]_{q}} D_{q}\left(\pi_{p-1, n}(q ; z)\right) \\
& +\frac{q^{p-1}[n]_{q} z+[p-1]_{q}}{[n+p+1]_{q}}\left(\pi_{p-1, n}(q ; z)-e_{p-1}(z)\right) \\
& +\frac{q^{p-1}[n]_{q} z+[p-1]_{q}}{[n+p+1]_{q}} z^{p-1}-z^{p} \\
& +\frac{q^{p-1} z(1-z)}{[n+p+1]_{q}} D_{q}\left(\pi_{p-1, n}(q ; z)\right) \\
& +\frac{q^{p-1}[n]_{q} z+[p-1]_{q}}{[n+p+1]_{q}}\left(\pi_{p-1, n}(q ; z)-e_{p-1}(z)\right) \\
& +\frac{[p-1]_{q}}{[n+p+1]_{q}} z^{p-1}+\frac{q^{p-1}[n]_{q}-[n+p+1]_{q}}{[n+p+1]_{q}} z^{p} .
\end{aligned}
$$

However

$$
\begin{aligned}
\left|\frac{q^{p-1}[n]_{q}-[n+p+1]_{q}}{[n+p+1]_{q}} z^{p}\right|=\mid & \left|\frac{q^{p-1}[n]_{q}-[p-1]_{q}-q^{p-1}[n]_{q}-q^{n+p-1}-q^{n+p}}{[n+p+1]_{q}} z^{p}\right| \\
& \leq \frac{[p+1]_{q}}{[n+p+1]_{q}} r^{p} .
\end{aligned}
$$


Combining the above relations and inequalities, we find

$$
\begin{aligned}
\left|\pi_{p, n}(q ; z)-e_{p}(z)\right| \leq & \frac{r(1+r)}{[n+2]_{q}} \cdot \frac{p-1}{r}\left\|\pi_{p-1, n}(q ; z)\right\|_{r} \\
& +r\left|\pi_{p-1, n}(q ; z)-e_{p-1}(z)\right|+\frac{[p+1]_{q}}{[n+2]_{q}} r^{p-1}(1+r) \\
& \leq \frac{(1+r)(p-1)}{[n+2]_{q}} r^{p-1}+r\left|\pi_{p-1, n}(q ; z)-e_{p-1}(z)\right| \\
& +\frac{[p+1]_{q}}{[n+2]_{q}} r^{p-1}(1+r) \\
& \leq 2 p \frac{(1+r)}{[n+2]_{q}} r^{p-1}+r\left|\pi_{p-1, n}(q ; z)-e_{p-1}(z)\right| .
\end{aligned}
$$

From the last inequality, inductively it follows that

$$
\begin{aligned}
\left|\pi_{p, n}(q ; z)-e_{p}(z)\right| \leq r( & \left.r\left|\pi_{p-2, n}(q ; z)-e_{p-2}(z)\right|+\frac{2(p-1)}{[n+2]_{q}}(1+r) r^{p-2}\right) \\
& +2 p \frac{(1+r)}{[n+2]_{q}} r^{p-1} \\
& =r^{2}\left|\pi_{p-2, n}(q ; z)-e_{p-2}(z)\right|+2 \frac{(1+r)}{[n+2]_{q}} r^{p-1}(p-1+p) \\
& \leq \cdots \leq \frac{(1+r)}{[n+2]_{q}} p(p+1) r^{p-1} .
\end{aligned}
$$

Thus, we obtain

$$
\left|M_{n, q}(f ; z)-f(z)\right| \leq \sum_{p=1}^{\infty}\left|a_{p}\right| \cdot\left|\pi_{p, n}(q ; z)-e_{p}(z)\right| \leq \frac{1+r}{[n+2]_{q}} \sum_{p=1}^{\infty}\left|a_{p}\right| p(p+1) r^{p-1} .
$$

which proves the theorem.

Remark 1. Let $q \in(0,1)$ be fixed. Since, $\lim _{n \rightarrow \infty} \frac{1}{[n+2]_{q}}=1-q$. Theorem 1, is not a convergence result. To obtain the convergence one can choose $0<q_{n}<1$ with $q_{n}>1$ as $n$ $\rightarrow \infty$. In that case $\frac{1}{[n+2]_{q_{n}}} \rightarrow 0$ as $n \rightarrow \infty($ see Videnskii [9], formula(2.7)), from Theorem 1 we get $M_{n, q_{n}}(f ; z) \rightarrow f(z)$, uniformly for $|z| \leq r$, and for any $1 \leq r<R$.

\section{Asymptotic formula and exact order}

Here we shall present the following quantitative Voronovskaja-type asymptotic result:

Theorem 2. Suppose that $f \in H\left(D_{R}\right), R>1$. Then, for any fixed $r \in[1, R]$ and for all $n \in \mathbb{N}, \quad|z| \leq r$ and $q \in(0, \quad 1)$, we have $\left|M_{n, q}(f ; z)-f(z)-\frac{z(1-z) f^{\prime \prime}(z)-2 z f^{\prime}(z)}{[n]_{q}}\right| \leq \frac{M_{r}(f)}{[n]_{q}^{2}}+2(1-q) \sum_{k=1}^{\infty}\left|a_{k}\right| k r^{k}$,

where $M_{r}(f)=\sum_{k=1}^{\infty}\left|a_{k}\right| k B_{k, r} r^{k}<\infty$, and

$$
B_{k, r}=(k-1)(k-2)(2 k-3)+8 k(k-1)^{2}+6(k-1) k^{2}++4 k(k-1)^{2}(1+r) .
$$


Proof. In view of the proof of Theorem 1, we can write $M_{n, q}(f ; z)=\sum_{k=0}^{\infty} a_{k} \pi_{k, n}(q ; z)$. Thus

$$
\begin{aligned}
& \left|M_{n, q}(f ; z)-f(z)-\frac{z(1-z) f^{\prime \prime}(z)-2 z f^{\prime}(z)}{[n]_{q}}\right| \\
& \leq \sum_{k=1}^{\infty}\left|a_{k}\right|\left|\pi_{k, n}(q ; z)-e_{k}(z)-\frac{(k(k-1)-k(k+1) z) z^{k-1}}{[n]_{q}}\right|,
\end{aligned}
$$

for all $z \in D_{R}, n \in \mathbb{N}$. If we denote

$$
E_{k, n}(q ; z)=\pi_{k, n}(q ; z)-e_{k}(z)-\frac{(k(k-1)-k(k+1) z) z^{k-1}}{[n]_{q}},
$$

then $E_{k, n}(q ; z)$ is a polynomial of degree $\leq k$, and by simple calculation and using Lemma 3, we have

$$
E_{k, n}(q ; z)=\frac{q^{k-1} z(1-z)}{[n+k+1]_{q}} D_{q} E_{k-1, n}(q ; z)+\frac{q^{k-1}[n]_{q} z+[k-1]_{q}}{[n+k+1]_{q}} E_{k-1, n}(q ; z)+X_{k, n}(q ; z),
$$

where

$$
\begin{aligned}
X_{k, n}(q ; z) & =\frac{z^{k-2}}{[n]_{q}[n+k+1]_{q}}\left[q^{k-1}(k-1)(k-2)[k-2]_{q}+[k-1]_{q}(k-1)(k-2)\right. \\
& +z\left(q^{k-1}[n]_{q}[k-1]_{q}-q^{k-1}(k-1)(k-2)[k-2]_{q}-q^{k-1} k(k-1)[k-1]_{q}\right. \\
& \left.+q^{k-1}[n]_{q}(k-1)(k-2)+[k-1]_{q}[n]_{q}-[k-1]_{q} k(k-1)-k(k-1)[n+k+1]_{q}\right) \\
& z^{2}\left(k(k+1)[n+k+1]_{q}-[n]_{q}[n+k+1]_{q}-q^{k-1}[n]_{q} k(k-1)\right. \\
& \left.\left.+q^{k-1}[n]_{q}^{2}+q^{k-1} k(k-1)[k-1]_{q}-q^{k-1}[n]_{q}[k-1]_{q}\right)\right] \\
& =: \frac{z^{k-2}}{[n]_{q}[n+k+1]_{q}}\left(X_{1, q, n}(k)+z X_{2, q, n}(k)+z^{2} X_{3, q, n}(k)\right) .
\end{aligned}
$$

Obviously as $0<q<1$, it follows that

$$
\left|X_{1, q, n}(k)\right| \leq(k-1)(k-2)(2 k-3) .
$$

Next with $[n+k+1]_{q}=[k-1]_{q}+q^{k-1}[n]_{q}+q^{n+k-1}+q^{n+k}$, we have

$$
\begin{aligned}
X_{2, q, n}(k) & =[n]_{q}\left(q^{k-1}[k-1]_{q}+[k-1]_{q}-2 q^{k-1}(k-1)\right) \\
& -q^{k-1}(k-1)(k-2)[k-2]_{q}-q^{k-1} k(k-1)[k-1]_{q} \\
& -[k-1]_{q} k(k-1)-k(k-1)[k-1]_{q}-k(k-1) q^{n+k-1}-k(k-1) q^{n+k}
\end{aligned}
$$

and

$$
\begin{aligned}
& {[n]_{q}\left(q^{k-1}[k-1]_{q}+[k-1]_{q}-2 q^{k-1}(k-1)\right)} \\
& =[n]_{q} q^{k-1}\left([k-1]_{q}-(k-1)\right)+[n]_{q}\left([k-1]_{q}-q^{k-1}(k-1)\right) \\
& =[n]_{q} q^{k-1}(q-1) \sum_{j=0}^{k-2}[j]_{q}+[n]_{q}(1-q) \sum_{j=1}^{k-1}[j]_{q} q^{k-1-j} \\
& =q^{k-1}\left(q^{n}-1\right) \sum_{j=0}^{k-2}[j]_{q}+\left(1-q^{n}\right) \sum_{j=1}^{k-1}[j]_{q} q^{k-1-j}
\end{aligned}
$$


Thus,

$$
\begin{aligned}
\left|X_{2, q, n}(k)\right| & \leq(k-1)[k-2]_{q}+(k-1)[k-1]_{q} \\
& +(k-1)(k-2)[k-2]_{q}+k(k-1)[k-1]_{q}+[k-1]_{q} k(k-1) \\
& +k(k-1)[k-1]_{q}+k(k-1)+k(k-1) \\
& \leq 8 k(k-1)^{2} .
\end{aligned}
$$

Now we will estimate $X_{3, q, n}(k)$ :

$$
\begin{aligned}
X_{3, q, n}(k)= & k(k+1)[n+k+1]_{q}-[n]_{q}[n+k+1]_{q}-q^{k-1}[n]_{q} k(k-1) \\
& +q^{k-1}[n]_{q}^{2}+q^{k-1} k(k-1)[k-1]_{q}-q^{k-1}[n]_{q}[k-1]_{q} \\
& =k(k+1)\left([k-1]_{q}+q^{k-1}[n]_{q}+q^{n+k-1}+q^{n+k}\right) \\
& -[n]_{q}\left([k-1]_{q}+q^{k-1}[n]_{q}+q^{n+k-1}+q^{n+k}\right)-q^{k-1}[n]_{q} k(k-1) \\
& +q^{k-1}[n]_{q}^{2}+q^{k-1} k(k-1)[k-1]_{q}-q^{k-1}[n]_{q}[k-1]_{q} \\
& =k(k+1)[k-1]_{q}+k(k+1)\left(q^{n+k-1}+q^{n+k}\right)-[n]_{q}[k-1]_{q} \\
& -[n]_{q}\left(q^{n+k-1}+q^{n+k}\right)+2 k q^{k-1}[n]_{q} \\
& +q^{k-1} k(k-1)[k-1]_{q}-q^{k-1}[n]_{q}[k-1]_{q} \\
& =[n]_{q}\left(-q^{k-1}[k-1]_{q}-[k-1]_{q}+q^{k-1}(2 k)-q^{n+k-1}-q^{n+k}\right) \\
& +k(k+1)[k-1]_{q}+k(k+1)\left(q^{n+k-1}+q^{n+k}\right)+q^{k-1} k(k-1)[k-1]_{q} \\
& =-[n]_{q} q^{k-1}\left([k-1]_{q}-(k-1)\right)+[n]_{q} q^{k-1}\left(1-q^{n}\right)+[n]_{q}\left(k q^{k-1}-[k-1]_{q}-q^{n+k}\right) \\
& +k(k+1)[k-1]_{q}+k(k+1)\left(q^{n+k-1}+q^{n+k}\right)+q^{k-1} k(k-1)[k-1]_{q} \\
= & -[n]_{q} q^{k-1}\left([k-1]_{q}-(k-1)\right)+[n]_{q} q^{k-1}\left(1-q^{n}\right)-[n]_{q}\left([k-1]_{q}-(k-1) q^{k-1}\right) \\
- & {[n]_{q}\left(q^{n+k}-q^{k-1}\right)+k(k+1)[k-1]_{q}+k(k+1)\left(q^{n+k-1}+q^{n+k}\right)+q^{k-1} k(k-1)[k-1]_{q} } \\
= & -q^{k-1}\left(q^{n}-1\right) \sum_{j=0}^{k-2}[j]_{q}-\left(1-q^{n}\right) \sum_{j=1}^{k-1}[j]_{q} q^{k-1-j}+q^{k-1}\left(1-q^{n}\right)[n]_{q} \\
- & {[n]_{q}\left(q^{n+k}-q^{k-1}\right)+k(k+1)[k-1]_{q}+k(k+1)\left(q^{n+k-1}+q^{n+k}\right)+q^{k-1} k(k-1)[k-1]_{q} . }
\end{aligned}
$$

Hence, it follows that

$$
\begin{aligned}
\left|X_{3, q, n}(k)\right| & \leq(k-1)[k-2]_{q}+(k-1)[k-1]_{q}+\left(1-q^{n}\right)[n]_{q} \\
& +\left(1-q^{n+1}\right)[n]_{q}+k(k+1)[k-1]_{q}+2 k(k+1)+k(k-1)[k-1]_{q} \\
& \leq 6(k-1) k^{2}+\left(1-q^{n}\right)[n]_{q}+\left(1-q^{n+1}\right)[n]_{q} .
\end{aligned}
$$

Thus,

$$
\begin{aligned}
\left|X_{k, n}(q ; z)\right| \leq & \frac{r^{k-2}}{[n]_{q}^{2}}\left((k-1)(k-2)(2 k-3)+r 8 k(k-1)^{2}+r^{2} 6(k-1) k^{2}\right) \\
& +\frac{r^{k}}{[n]_{q}}\left(1-q^{n}\right)+\frac{r^{k}}{[n+1]_{q}}\left(1-q^{n+1}\right) \\
& =\frac{r^{k-2}}{[n]_{q}^{2}}\left((k-1)(k-2)(2 k-3)+r 8 k(k-1)^{2}+r^{2} 6(k-1) k^{2}\right)+2 r^{k}(1-q)
\end{aligned}
$$

for all $k \geq 1, n \in \mathbb{N}$ and $|z| \leq r$.

Next, using the estimate in the proof of Theorem 1, we have

$$
\left|\pi_{k, n}(q ; z)-e_{k}(z)\right| \leq \frac{(1+r) k(k+1) r^{k-1}}{[n+2]_{q}}
$$

for all $k, n \in \mathbb{N},|z| \leq r$, with $1 \leq r$. 
Hence, for all $k, n \in \mathbb{N}, k \geq 1$ and $|z| \leq r$, we have

$$
\left|E_{k, n}(q ; z)\right| \leq \frac{q^{k-1} r(1+r)}{[n+k+1]_{q}}\left|E_{k-1 n}^{\prime}(q ; z)\right|+\frac{q^{k-1}[n]_{q} r+[k-1]_{q}}{[n+k+1]_{q}}\left|E_{k-1, n}(q ; z)\right|+\left|X_{k, n}(q ; z)\right| .
$$

However, since $\frac{q^{k-1} r(1+r)}{[n+k+1]_{q}} \leq \frac{r(1+r)}{[n+k+1]_{q}}$ and $\frac{q^{k-1}[n]_{q} r+[k-1]_{q}}{[n+k+1]_{q}} r$, it follows that

$$
\left|E_{k, n}(q ; z)\right| \leq \frac{r(1+r)}{[n+k+1]_{q}}\left|E_{k-1, n}^{\prime}(q ; z)\right|+r\left|E_{k-1, n}(q ; z)\right|+\left|X_{k, n}(q ; z)\right| .
$$

Now we shall compute an estimate for $-E_{k-1, n}^{\prime}(q ; z)-, k \geq 1$. For this, taking into account the fact that $E_{k-1, n}(q ; z)$ is a polynomial of degree $\leq k-1$, we have

$$
\begin{aligned}
& \left|E_{k-1, n}^{\prime}(q ; z)\right| \leq \frac{k-1}{r}\left\|E_{k-1, n}\right\|_{r} \\
\leq & \frac{k-1}{r}\left[\left\|\pi_{k-1, n}-e_{k-1}\right\|_{r}+\left\|\frac{\left.\left\{(k-1)(k-2)-k(k-1) e_{1}\right\} e_{k-2}\right]}{[n]_{q}}\right\|_{r}\right] \\
\leq & \frac{k(k-1)}{r}\left[\frac{(1+r)(k-1) k r^{k-2}}{[n+2]_{q}}+\frac{r^{k-2} k(k-1)(1+r)}{[n]_{q}}\right] \\
\leq & \frac{k(k-1)^{2}}{[n]_{q}}\left[2 r^{k-2}+2 r^{k-2}\right]=\frac{4 k(k-1)^{2} r^{k-2}}{[n]_{q}}
\end{aligned}
$$

Thus,

$$
\frac{r(1+r)}{[n+k+1]_{q}}\left|E_{k-1, n}^{\prime}(q ; z)\right| \leq \frac{4 k(k-1)^{2}(1+r) r^{k-1}}{[n]_{q}^{2}}
$$

and

$$
\left|E_{k, n}(q ; z)\right| \leq \frac{4 k(k-1)^{2}(1+r) r^{k}}{[n]_{q}^{2}}+r\left|E_{k-1, n}(q ; z)\right|+\left|X_{k, n}(q ; z)\right|,
$$

where

$$
\left|X_{k, n}(q ; z)\right| \leq \frac{r^{k}}{[n]_{q}^{2}} A_{k}+2 r^{k}(1-q),
$$

for all $|z| \leq r, k \geq 1, n \in \mathbb{N}$, where

$$
A_{k}=(k-1)(k-2)(2 k-3)+8 k(k-1)^{2}+6(k-1) k^{2} .
$$

Hence, for all $|z| \leq r, k \geq 1, n \in \mathbb{N}$

$$
\left|E_{k, n}(q ; z)\right| \leq r\left|E_{k-1, n}(q ; z)\right|+\frac{r^{k}}{[n]_{q}^{2}} B_{k, r}+2 r^{k}(1-q),
$$

where $B_{k, r}$ is a polynomial of degree 3 in $k$ defined as

$$
B_{k, r}=A_{k}+4 k(k-1)^{2}(1+r) .
$$


But $E_{0, n}(q ; z)=0$, for any $z \in C$, and therefore by writing the last inequality for $k=$ $1,2, \ldots$ we easily obtain step by step the following

$$
\left|E_{k, n}(q ; z)\right| \leq \frac{r^{k}}{[n]_{q}^{2}} \sum_{j=1}^{k} B_{j, r}+2 r^{k}(1-q) \leq \frac{k r^{k}}{[n]_{q}^{2}} B_{k, r}+2 r^{k} k(1-q) .
$$

Therefore, we can conclude that

$$
\begin{aligned}
& \left|M_{n, q}(f ; z)-f(z)-\frac{z(1-z) f^{\prime \prime}(z)-2 z f^{\prime}(z)}{[n]_{q}}\right| \leq \sum_{k=1}^{\infty}\left|a_{k}\right|\left|E_{k, n}(q ; z)\right| \\
& \quad \leq \frac{1}{[n]_{q}^{2}} \sum_{k=1}^{\infty}\left|a_{k}\right| k B_{k, r} r^{k}+2(1-q) \sum_{k=1}^{\infty}\left|a_{k}\right| k r^{k} .
\end{aligned}
$$

$\operatorname{As} f^{(4)}(z)=\sum_{k=4}^{\infty} a_{k} k(k-1)(k-2)(k-3) z^{k-4}$ and the series is absolutely convergent in $|z| \leq r$, it easily follows that $\sum_{k=4}^{\infty}\left|a_{k}\right| k(k-1)(k-2)(k-3) r^{k-4}<\infty$, which implies that $\sum_{k=1}^{\infty}\left|a_{k}\right| k B_{k, r} r^{k}<\infty$. This completes the proof of theorem.

Remark 2. For $q \in(0,1)$ fixed, we have $\frac{1}{[n]_{q}} \rightarrow 1-q$ as $n \rightarrow \infty$, thus Theorem 2 does not provide convergence. But this can be improved by choosing $1-\frac{1}{n^{2}} \leq q_{n}<1$ with $q_{n}$ $\nearrow 1$ as $n \rightarrow \infty$. Indeed, since in this case $\frac{1}{[n]_{q_{n}}} \rightarrow 0$ as $n \rightarrow \infty$ and $1-q_{n} \leq \frac{1}{n^{2}} \leq \frac{1}{[n]_{q_{n}}^{2}}$ from Theorem 2, we get

$$
\left|M_{n, q_{n}}(f ; z)-f(z)-\frac{z(1-z) f^{\prime \prime}(z)-2 z f^{\prime}(z)}{[n]_{q_{n}}}\right| \leq \frac{M_{r}(f)}{[n]_{q_{n}}^{2}}+\frac{2}{[n]_{q_{n}}^{2}} \sum_{k=1}^{\infty}\left|a_{k}\right| k r^{k} .
$$

Our next main result is the exact order of approximation for the operator (1.1).

Theorem 3. Let $1-\frac{1}{n^{2}} \leq q_{n}<1, n \in \mathbb{N}, R>1$, and let $f \in H\left(D_{R}\right), R>1$. If $f$ is not a polynomial of degree 0 , then for any $r \in[1, R)$, we have

$$
\left\|M_{n, q_{n}}(f ; \cdot)-f\right\|_{r} \geq \frac{C_{r}(f)}{[n]_{q_{n}}}, n \in \mathbb{N},
$$

where the constant $C_{r}(f)>0$ depends on $f, r$ and on the sequence $\left(q_{n}\right)_{n \in \mathbb{N}}$, but it is inde-pendent of $n$.

Proof. For all $z \in \mathbb{D}_{R}$ and $n \in \mathbb{N}$, we have

$$
\begin{gathered}
M_{n, q_{n}}(f ; z)-f(z)=\frac{1}{[n]_{q_{n}}}\left[z(1-z) f^{\prime \prime}(z)-2 z f^{\prime}(z)\right. \\
\left.+\frac{1}{[n]_{q_{n}}}\left\{[n]_{q_{n}}^{2}\left(M_{n, q_{n}}(f ; z)-f(z)-\frac{z(1-z) f^{\prime \prime}(z)-2 z f^{\prime}(z)}{[n]_{q_{n}}}\right)\right\}\right] .
\end{gathered}
$$

We use the following property.

$$
\|F+G\|_{r} \geq\left\||| F||_{r}-\right\| G\left\|_{r} \mid \geq\right\| F\left\|_{r}-\right\| G \|_{r} .
$$


to obtain

$$
\begin{gathered}
\left\|M_{n, q_{n}}(f ; \cdot)-f\right\|_{r} \geq \frac{1}{[n]_{q_{n}}}\left[\left\|e_{1}\left(1-e_{1}\right) f^{\prime \prime}-2 e_{1} f^{\prime}\right\|_{r}\right. \\
\left.-\frac{1}{[n]_{q_{n}}}\left\{[n]_{q_{n}}^{2}\left\|M_{n, q_{n}}(f ; \cdot)-f-\frac{e_{1}\left(1-e_{1}\right) f^{\prime \prime}-2 e_{1} f^{\prime}}{[n]_{q_{n}}}\right\|_{r}\right\}\right] .
\end{gathered}
$$

By the hypothesis, $f$ is not a polynomial of degree 0 in $D_{R}$, we get $\left\|e_{1}\left(1-e_{1}\right) f^{\prime \prime}-2 e_{1} f\right\|_{r}$ $>0$. Supposing the contrary, it follows that $z(1-z) f^{\prime \prime}(z)-2 z f(z)=0$ for all $|z| \leq r$, that is $(1-z) f^{\prime \prime}(z)-2 f(z)=0$ for all $|z| \leq r$ with $z \neq 0$. The last equality is equivalent to [( 1 - z) $f(z)]^{\prime}-f(z)=0$, for all $|z| \leq r$ with $z \neq 0$. Therefore, $(1-z) f(z)-f(z)=C$, where $C$ is a constant, that is, $f(z)=\frac{C_{z}}{1-z}$, for all $|z| \leq r$ with $z \neq 0$. But since $f$ is analytic in $\bar{D}_{r}$ and $r \geq 1$, we necessarily have $C=0$, a contradiction to the hypothesis.

But by Remark 2, we have

$$
[n]_{q_{n}}^{2}\left\|M_{n, q_{n}}\left(f_{;} \cdot\right)-f-\frac{e_{1}\left(1-e_{1}\right) f^{\prime \prime}-2 e_{1} f^{\prime}}{[n]_{q_{n}}}\right\|_{r} \leq M_{r}(f)+2 \sum_{k=1}^{\infty}\left|a_{k}\right| k r^{k},
$$

with $\frac{1}{[n]_{q_{n}}} \rightarrow 0$ as $n \rightarrow \infty$. Therefore, it follows that there exists an index $n_{0}$ depending only on $f, r$ and on the sequence $\left(q_{n}\right)_{n}$, such that for all $n \geq n_{0}$, we have

$$
\begin{gathered}
\left\|e_{1}\left(1-e_{1}\right) f^{\prime \prime}-2 e_{1} f^{\prime}\right\|_{r} \\
-\frac{1}{[n]_{q_{n}}}\left\{[n]_{q_{n}}^{2}\left\|M_{n, q_{n}}(f ; \cdot)-f-\frac{e_{1}\left(1-e_{1}\right) f^{\prime \prime}-2 e_{1} f^{\prime}}{[n]_{q_{n}}}\right\|_{r}\right\} \\
\geq \frac{1}{2}\left\|e_{1}\left(1-e_{1}\right) f^{\prime \prime}-2 e_{1} f^{\prime}\right\|_{r}
\end{gathered}
$$

which implies that

$$
\left\|M_{n, q_{n}}(f ; \cdot)-f\right\|_{r} \geq \frac{1}{2[n]_{q_{n}}}\left\|e_{1}\left(1-e_{1}\right) f^{\prime \prime}-2 e_{1} f^{\prime}\right\|_{r}, \forall n \geq n_{0} .
$$

For $1 \leq n \leq n_{0}-1$, we clearly have

$$
\left\|M_{n, q_{n}}(f ; \cdot)-f\right\|_{r} \geq \frac{c_{r, n}(f)}{[n]_{q_{n}}}
$$

where $c_{r, n}(f)=[n]_{q_{n}} \cdot\left\|M_{n, q_{n}}(f ; \cdot)-f\right\|_{r}>0$, which finally implies

$$
\left\|M_{n, q_{n}}(f ; \cdot)-f\right\|_{r} \geq \frac{C_{r}(f)}{[n]_{q_{n}}}, \text { for all } n \in \mathbb{N},
$$

where

$$
C_{r}(f)=\min \left\{c_{r, 1}(f), c_{r, 2}(f), \ldots, c_{r, n_{0}-1}(f), \frac{1}{2}\left\|e_{1}\left(1-e_{1}\right) f^{\prime \prime}-2 e_{1} f^{\prime}\right\|_{r}\right\} .
$$

$\square$

Combining Theorem 3 with Theorem 1 we get the following. 
Corollary 2. Let $1-\frac{1}{n^{2}}<q_{n}<1$ for all $n \in \mathbb{N}, R>1$, and suppose that $f \in H\left(D_{R}\right)$. If $f$ is not a polynomial of degree 0 , then for any $r \in[1, R)$, we have

$$
\left\|M_{n, q_{n}}(f ; \cdot)-f\right\|_{r} \sim \frac{1}{[n]_{q_{n}}}, n \in \mathbb{N},
$$

where the constants in the above equivalence depend on $f, r,\left(q_{n}\right)_{n}$, but are independent of $n$.

The proof follows along the lines of [7].

Remark 3. For $0 \leq \alpha \leq \beta$, we can define the Stancu type generalization of the operators (1.1) as

$$
M_{n, q}^{\alpha, \beta}(f ; z)=[n+1]_{q} \sum_{k=1}^{n} q^{1-k} p_{n, k}(q ; z) \int_{0}^{1} f\left(\frac{[n]_{q} t+\alpha}{[n]_{q}+\beta}\right) p_{n, k-1}(q ; q t) d_{q} t+f\left(\frac{\alpha}{[n]_{q}+\beta}\right) p_{n, 0}(q ; z) .
$$

The analogous results can be obtained for such operators. As analysis is different, it may be considered elsewhere.

\section{Acknowledgements}

The authors are thankful to the referees for valuable suggestions leading to overall improvements in article.

\section{Author details}

'Department of Mathematics, Texas A\&M University-Kingsville, 700 University Blvd., Kingsville, TX 78363-8202, USA

${ }^{2}$ School of Applied Sciences, Netaji Subhas Institute of Technology, Sector 3 Dwarka, New Delhi-110078, India

\section{Authors' contributions}

All authors read and approved the final manuscript.

\section{Competing interests}

The authors declare that they have no competing interests.

Received: 17 February 2012 Accepted: 22 May 2012 Published: 22 May 2012

\section{References}

1. Kim, $\mathrm{T}$ : Some Identities on the $q$-integral representation of the product of several $q$-Bernstein-type polynomials, abstract and applied analysis. (2011) Article ID 634675, 11 (2011). doi:10.1155/2011/634675

2. Kim, T: q-generalized Euler numbers and polynomials. Russ J Math Phys. 13(3):293-298 (2006). doi:10.1134/ S1061920806030058

3. Gupta, V, Wang, H: The rate of convergence of $q$-Durrmeyer operators for $0<q<1$. Math Meth Appl Sci. 31, 1946-1955 (2008). doi:10.1002/mma.1012

4. Gal, SG: Approximation by Complex Bernstein and Convolution-Type Operators. World Scientific Publ Co, Singapore. (2009)

5. Gal, SG, Gupta, V: Approximation by a Durrmeyer-type operator in compact disk. Annali Dell Universita di Ferrara. 57, 261-274 (2011). doi:10.1007/s11565-011-0124-6

6. Gal, SG, Gupta, V: Quantative estimates for a new complex Durrmeyer operator in compact disks. Appl Math Comput. 218(6):2944-2951 (2011)

7. Gal, S, Gupta, V, Mahmudov, NI: Approximation by a complex q Durrmeyer type operators. Ann Univ Ferrara. 58(1):65-82 (2012). doi:10.1007/s11565-012-0147-7

8. Kac, V, Cheung, P: Quantum Calculus. Springer, New York (2002)

9. Videnskii, VS: On q-Bernstein polynomials and related positive linear operators (in Russian). Problems of Modern Mathematics and Mathematical Education, St.-Petersburg. 118-126 (2004)

10. Gupta, V, Yadav, R: Approximation by a complex summation-integral type operator in compact disks. Mathematica Slovaca (to appear)

doi:10.1186/1029-242X-2012-111

Cite this article as: Agarwal and Gupta: On $q$-analogue of a complex summation-integral type operators in compact disks. Journal of Inequalities and Applications 2012 2012:111. 